\title{
Nonlinear Gust Response Analysis of Free Flexible Aircraft
}

\author{
Guo Dong, Xu Min, Chen Shilu \\ College of Astronautics, Northwestern Polytechnical University, Xi'an, China \\ guodong@mail.nwpu.edu.cn; xumin@nwpu.edu.cn
}

\begin{abstract}
Gust response analysis plays a very important role in large aircraft design. This paper presents a methodology for calculating the flight dynamic characteristics and gust response of free flexible aircraft. A multidisciplinary coupled numerical tool is developed to simulate detailed aircraft models undergoing arbitrary free flight motion in the time domain, by Computational Fluid Dynamics (CFD), Computational Structure Dynamics (CSD) and Computational Flight Mechanics (CFM) coupling. To achieve this objective, a structured, time-accurate flowsolver is coupled with a computational module solving the flight mechanics equations of motion and a structural mechanics code determining the structural deformations. A novel method to determine the trim state of flexible aircraft is also stated. First, the field velocity approach is validated, after the trim state is attained, gust responses for the one-minus-cosine gust profile are analyzed for the longitudinal motion of a slender-wing airc raft configuration with and without the consideration of structural deformation.
\end{abstract}

Index Terms - Numerical Simulation, Computational Flight Mechanics, Multi-disciplinary Coupled, Gust Response

\section{Introduction}

Modern aircraft design requires the evaluation of dynamic loads in response to discrete and random gust excitations. Gust response affects many aspects of aircraft characteristics, including stability and control, dynamic structural loads, flight safety and easement. The Federal Aviation Regulations require that the aircraft structure can withstand discrete gusts of certain profile, intensity and gradient [1]. Because of its multidisciplinary nature with aerodynamics, flight mechanics, aeroelasitcity, and atmospheric turbulence, until now, only the doublet-lattice, unsteady linear aerodynamic cod DLM, coupled with the equation of motion of flexible vehicle, was used for gust response analysis [2-5].

Flight mechanics and aeroelasticity can be often treated as separate discipline, namely, flight mechanics and aeroelasticity. The first one concerned principally with rigid aircraft experiencing large motions (commonly known as Rigid Body Approximation-
RBA), while the second aims mainly to the analysis of elastic aircraft experiencing relatively small deformations. The idea to not consider cross-coupling effects between these two disciplines has been commonly justified by the large frequency separation of the characteristic motion which is typical of conventional structures. Nowadays, the focus on weight minimization for aircraft, leads toward more and more flexible vehicles. The resulting underlying structures may not exhibit the usual wide frequency separation among the rigid body degrees of freedom and the remaining elastic modes. So that the approach mentioned above can lead to mistakes/errors in analyses of flight performance, flying qualities, and control systems design. In these cases, an integrated analys is of flight mechanics and aeroelasticity is necessary from the very early stages of preliminary design [6].

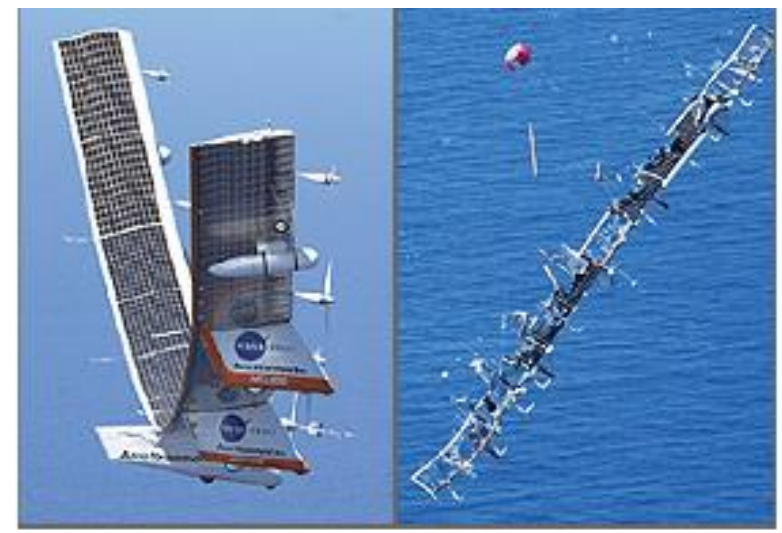

Fig. 1: HP03-2 mishap

Another reason which substantiates the development of an integrated approach is high-altitude; longendurance (HALE) unmanned aerial vehicles (UA Vs) are recently receiving considerable attention from the technical community. Patil [7], Shear [8], Cesnik and Weihua $\mathrm{Su}$ [9-10] showed that when the nonlinear flexibility effects are taken into account in the calculation of trim and flight dynamics characteristics, the predicted aeroelasitc behavior of the complete aircraft turns out to be very different from what it would be without such effects. The Helios accident also highlighted our limited understanding and limited analytical tools necessary for designing very flexible aircraft and to potentially exp loit airc raft fle xibility. The number one root cause/recommendation from NASA 
[11] was "more advanced, multidisciplinary (structures, aeroelastic, aerodynamics, atmospheric, materials, propulsion, controls, etc.) time-domain analysis methods appropriate to highly flexible, morphing vehicles [be developed]."

For all of the reasons stated, a better understanding of the flight dynamics/aeroelasticity of these vehicles is required. The key points are the aerodynamic loads as well as the mass distribution. During flight the maneuvers change the aerodynamic loads which act on the aircraft. Thus a variation on the structural displacements is expected, modifying the mass distribution and therefore the inertia moments, important parameters for the characterization of the aircraft dynamics. The structural displacements cause also a variation on the aerodynamic loads through the alteration of the body geometry and due to motion (its time derivative). Although there are commercial software tools capable of dealing with pieces of the problem, there is no commercially available software that integrates all of the disciplines needed for such investigation as discussed here.

The intention here is not to investigate complex flight mechanics behavior, but to describe the development of a tool which can be used for this purpose. This paper illustrates a multidisciplinary coupled numerical tool to simulate detailed aircraft models undergoing arbitrary free flight motion in the time domain, by Computational Fluid Dynamics (CFD), Computational Structure Dynamics (CSD) and Computational Flight Mechanics (CFM) coupling. A substantial part of this work is devoted to the development of an integrated aircraft flight mechanics model as generic as possible, which comprises the structure vibration influence, without loss of : the simplicity of the equations; the similarity to the classical RBA; and perhaps the most important, the physical understanding of the interactions. The modal decomposition technique is used to represent the structural dynamic [12]. The CFD solver developed by MFDCL (Multidisciplinary Flight Dynamic and Control Laboratory, College of Astronautics, Northwestern Polytechnical University), will be used to compute the unsteady aerodynamics loads in this work.

\section{Numerical Approach}

\subsection{Flight Model}

A finite element analysis using Patran/Nastran is assumed to have been performed, such that a modal description of the structure is available. That is, if the elastic deformation at position $(\mathrm{x}, \mathrm{y}, \mathrm{z})$ on the structure is denoted as $\mathbf{d}$, then it may be written as

$$
\mathbf{d}=\sum_{i=1}^{\infty} \phi_{i}(x, y, z) \eta_{i}
$$

where $\eta_{i}$ is a generalized coordinate of the structure and $\phi_{i}(x, y, z)$ is a mode shape. Of course, a finite number of modes must be used, so that the model always is based on a truncated-mode description.

The equations of motion are developed with the use of a special body-reference axis, the axis referred to by Milne as the mean axis. This axis is the coordinate frame with respect to which the elastic deformations contribute no translation or rotation mo mentu m. For the mean axis, the following relations hold:

$$
\int_{V} \frac{\delta \mathbf{p}}{\delta t} \rho d V=\int_{V} \mathbf{p} \times \frac{\delta \mathbf{p}}{\delta t} \rho d V=0
$$

where an infinitesimal volume of the vehicle $\mathrm{d} V$ has location $\mathbf{p}_{\text {with respect to the instantaneous center of }}$ mass of the vehicle. All of the equations of motion are derived using this body-referenced (mean) axis, and all forces, moments, and inertias must be resolved into components along and about the three directions, that is, $\mathrm{x}, \mathrm{y}$, and $\mathrm{z}$ of this axis.

The dynamic equations of motion for the elastic aircraft are then derived from first principles using Lagrange's equation [13]:

$$
\frac{d}{d t}\left(\frac{\partial T}{\partial \dot{q}_{i}}\right)-\frac{\partial T}{\partial q_{i}}+\frac{\partial U}{\partial q_{i}}=Q_{i}
$$

The resulting equations from the application of Eq. (3) may be expressed as follows:

Equations for rigid-body translational accelerations:

$$
\left\{\begin{array}{l}
M(\dot{u}+q w-r v+g \sin \theta)=X \\
M(\dot{v}+r u-p w-g \cos \theta \sin \phi)=Y \\
M(\dot{w}+p v-q u-g \cos \theta \cos \phi)=Z
\end{array}\right.
$$

Equations for rigid-body rotational accelerations:

$$
\begin{aligned}
& I_{x} \dot{p}-\left(I_{x y} \dot{q}+I_{x z} \dot{r}\right)+\left(I_{z}-I_{y}\right) q r+\left(I_{x y} r-I_{x z} q\right) p+\left(r^{2}-q^{2}\right) I_{y z}=L \\
& I_{y} \dot{q}-\left(I_{x y} \dot{p}+I_{y z} \dot{r}\right)+\left(I_{x}-I_{z}\right) p r+\left(I_{y z} p-I_{x y} r\right) q+\left(p^{2}-r^{2}\right) I_{x z}=M \\
& I_{z} \dot{r}-\left(I_{x z} \dot{p}+I_{y z} \dot{q}\right)+\left(I_{y}-I_{x}\right) p q+\left(I_{x z} q-I_{y z} p\right) r+\left(q^{2}-p^{2}\right) I_{x y}=N
\end{aligned}
$$


Equations for elastic degrees of freedom:

$$
\ddot{\eta}_{i}+2 \varsigma_{i} \omega_{i} \dot{\eta}_{i}+\omega_{i}^{2} \eta_{i}=Q_{\eta_{i}} / m_{i}
$$

\subsection{CFD Solver}

The behavior of the fluid flow affecting the object of interest is simulated with the EU3D-Code, a CFD tool developed by the Multidisciplinary Flight Dynamics and Control Laboratory. A full discussion of the code and turbulence models implemented is given in reference [14]. The EU3D-Code solves the compressible, three-dimensional, time-accurate Euler and RANS equations using a finite volume formulation. The Code is based on a structured-grid approach. The grids used for simulations in this paper were created with the grid generator Gridgen. The Code contains several upwind schemes, FDS-Roe, FVS-Vanleer, AUSM+ and AUSMpw+. LUSGS and dual time step marching scheme LUSGS- $\tau$ TS is also available.

\subsection{Coupled Solution Procedure}

The coupling flow chat is showed in Fig.2:

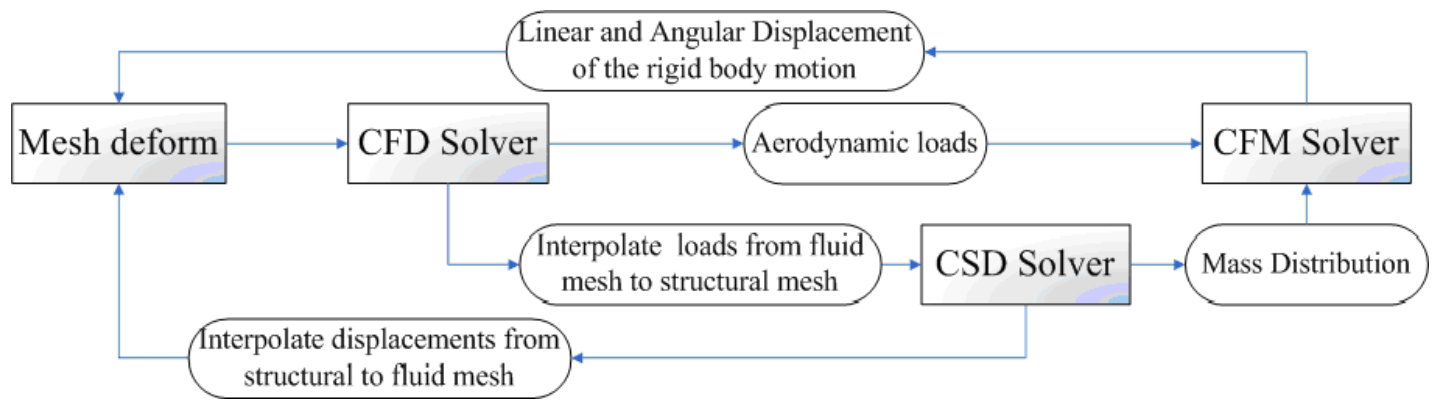

Fig. 2: CFD-based multidisciplinary coupled numerical simulation flow chart

The detail coupling algorithm is:

(a) The CFD solver computes a set of aerodynamic loads based on the mesh at a new time step;

(b) Interpolate aerodynamic loads from fluid mesh to structural mesh;

(c) The CSD solver uses the interpolated loads to compute the structure responses for the current time step, according to Equation (6);

(d) Compute the center of mass and the inertia tens or based on the mass distribution;

(e) The CFM solver uses the aerodynamic loads from the CFD solver and the mass characteristic to compute the flight dynamic response, according to Equation (4) and (5);

(f) Update the fluid mesh based on the structure and flight dynamic response, and send the updated mesh to the CFD solver;

(g) The loop goes back to point (a), until the desired condition is reached.

In the current implementation, the CFD solver takes care of computing the resultant forces and moments, and the generalized forces from the pressure distribution. When the CFD solver converges, it stops sending aerodynamic loads information and waiting for the updated fluid mesh information. The equations of flight dynamics and structure dynamics are solver by fourorder Runge-Kutta method.

CFD-CSD Interface. Generally, the CFD-CSD interface refers to the transformation to the structural grid of aerodynamic forces that are computed on the aerodynamic grid, and to the mapping of the elastic deformations computed on the structural finite element nodes to the aerodynamic grid. When the modal structural approach is taken, with the structure represented by a set of its low-frequency vibration nodes, the CFD-CSD interfacing task reduces to mapping the modes to the aerodynamic surface grid points. After this is done, the computation of elastic deformations can be carried on within the fluid dynamics computations. The Infin ite Plate Spline (IPS) method by Harder and Desmarais is applied to exchange the boundary information between the fluid mesh and the structure mesh [15]. Fig. 3 shows 30 times mode shapes mapped into CFD surface grid.

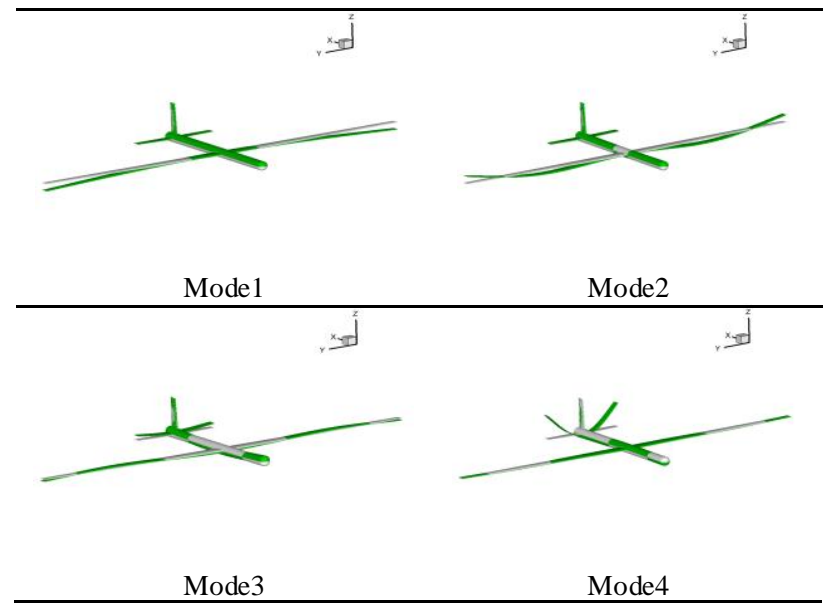

Fig.3: First four elast ic mode shapes mapped into CFD surface grid 
Aerodynamic Grid Update. The gird must be deformed once per time step in unsteady flow simulations. Once the aerodynamic surface grid points have been deflected to account for the control surface deflections and the rigid motion of the aircraft, the grids must also be deflected accordingly. A modified TFI method is employed here. The main ideas about the TFI method of grid perturbations are described in reference [14].

It should be noted that the longitudinal 3DOF equations are loosely coupled, in that they are solved sequentially for each time step. With the new CG locations and pitch attitude, the grid is rig idly translated and rotated accordingly, and the grid velocities are computed using second order finite differences. The process is illustrated in Fig. 4 for a generic aircraft test case with an all moving elevator. Fig. 5 shows that after the rotation of the aircraft, the flow girds were updated by TFI.

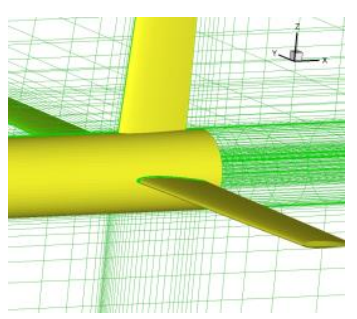

a) Original Surface Grid

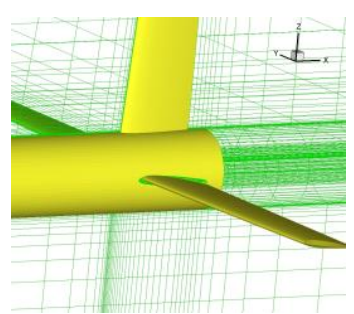

b) Elevator Deflected
Fig.4: Deflection of the elevator

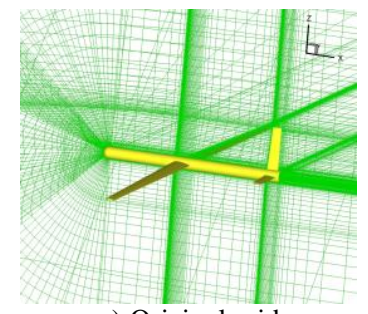

a) Original grid

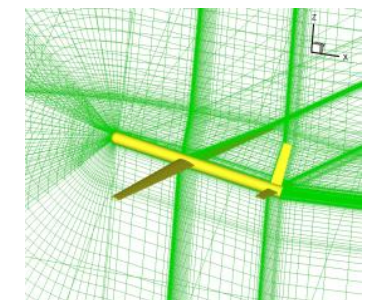

b) Rotated Grid (TFI)
Fig.5: Rotation of the aircraft

\subsection{Trim Process}

So far we have obtained a multid isciplinary coupled numerical tool to simulate the flexible aircraft in timedomain. The next step is generally necessary to first solve the so-called trim problem. For rigid body approximation, the trim problem can be characterized in the following way: find a set of suitable input values, e.g. aircraft angle of attack, elevator setting, thrust, to satisfy a set of conditions, e.g. to fly in a straight path in steady state, i.e. no accelerations along $x, Z$, and no rotational acceleration around $y$. For the elastic aircraft, an additional condition to the conventional trim requirements is that the elastic deformation is in steady state, i.e. the second derivatives of all body motion representing the elastic structure have to be zero [16].

In this case, the effect of thrust on the trim configuration is neglected and a non-null horizontal reaction appears. The aircraft is considered as trimmed when vertical reaction force and pitching moment are null. A deflection of the elevator is then used to guarantee the final equilibrium of vertical force and pitch moment. Fig. 6 shows the CFD mesh used for Euler calculations. The complete aircraft is modeled, despite symmetric flow conditions are considered in the following examples. An all-movable elevator is introduced in this case; the detail grid is outlined.

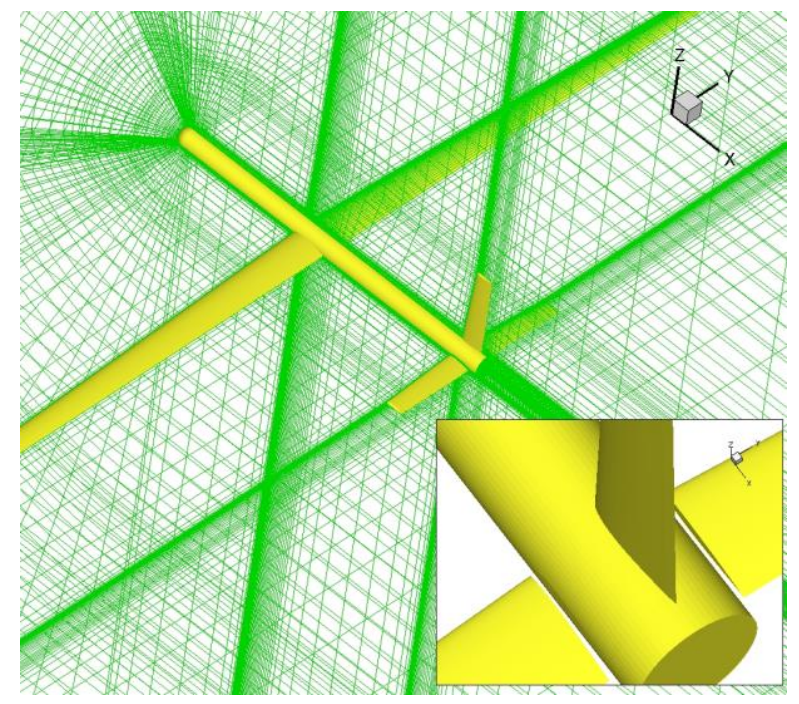

Fig. 6: The CFD mesh and elevator detail used for the numerical simulation

The solution of the trim problem is iteratively sought in a manner as outlined in Fig.7; it consists of three different nested iteration-levers [12]:

The Basic Level. Considering the aircraft is rigid, a maneuver trim loop adjusts elevator deflection and aircraft attitude to have the static equilibriu m position is sought. In this case, $M$ iterative loop is introduced to solve the Eq. (7) for symmetric maneuver.

$$
\left[\begin{array}{l}
\vartheta \\
\delta_{e}
\end{array}\right]^{n+1}=\left[\begin{array}{l}
\vartheta \\
\delta_{e}
\end{array}\right]^{n}+\left[\begin{array}{ll}
C_{Z}^{\vartheta} & C_{Z}^{\delta_{e}} \\
C_{M}^{\vartheta} & C_{M}^{\delta_{e}}
\end{array}\right]^{-1}\left[\begin{array}{l}
F_{z, r e s} \\
M_{y, r e s}
\end{array}\right]
$$

Where $F_{z, \text { res }}$ and $M_{y, \text { res }}$ refer to the residual of nondimensional flexible normal force and pitching moment terms that correspond to a current set of values of trim parameters. The value of $C_{i}^{\vartheta}, C_{i}^{\delta}(i=L, M)$ may be known experimentally or through the CFD solver. Including aeroelastic effects on these derivatives using the CFD solver leads to further computational costs. Thus for this simple application, their values are never updated and determined once for all starting from the undeformed reference condition of null pitch and elevator deflection. Of course, this leads to a slower convergence for trim corrections. 
The Medium Level. Once the $M$ iterations reached, assuming the variables giving the motion of the aircraft and its controls in the body reference frame as frozen, the deformed trim shape for the current configuration is sought. A user-defined nu mber of iterations $N$ is carried out:

$$
\mathbf{K} \boldsymbol{\eta}^{(i+1)}=\mathbf{Q}\left(\boldsymbol{\eta}^{(i)}, \mathbf{x}^{(n)}, \boldsymbol{\delta}^{(n)}\right)
$$

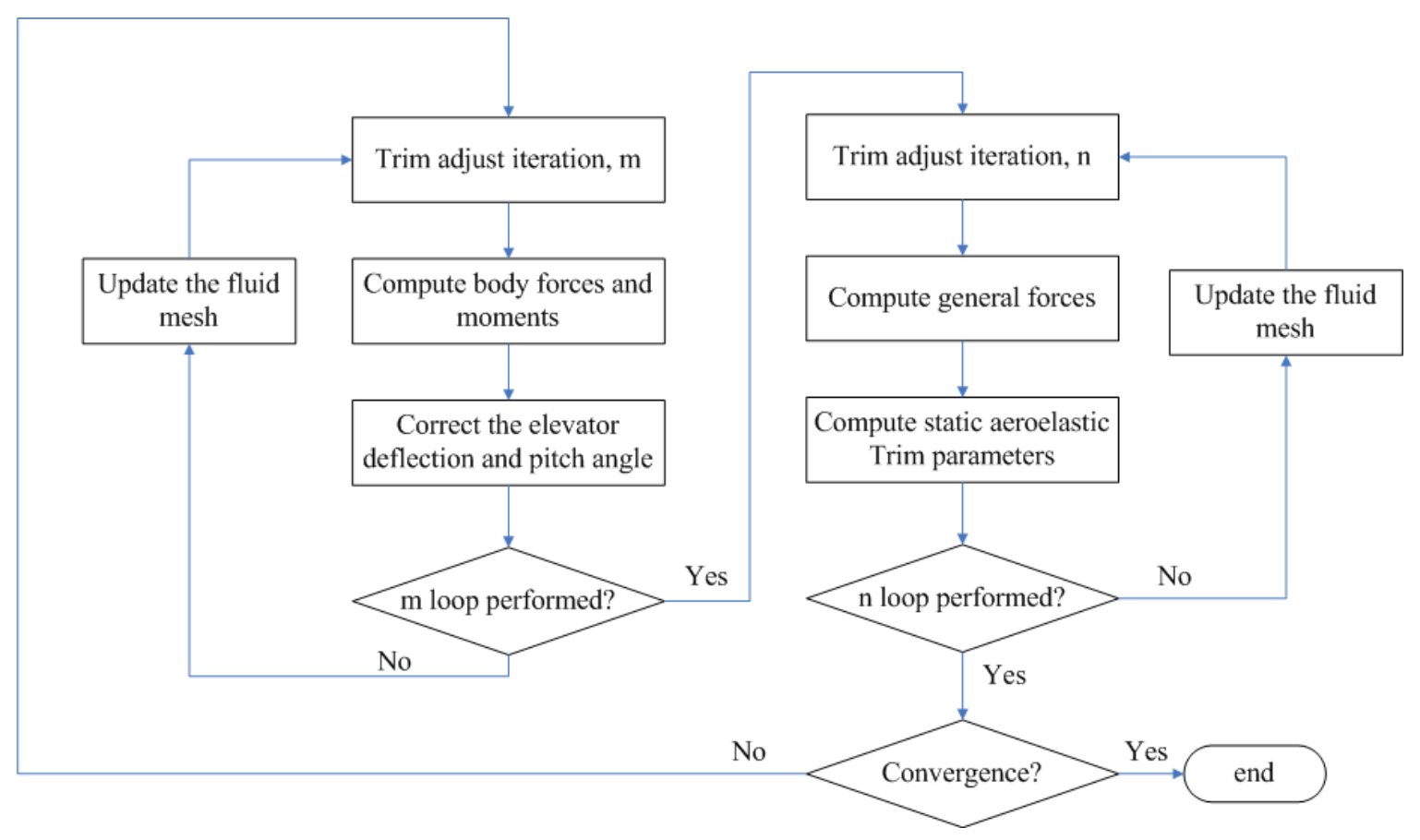

Fig.7: flow chart for the deformable trim process

\subsection{Field Velocity Method and Validations}

The so-called field velocity approach, developed by Baeder[17,18], is used to calculate the gust response. The field velocity approach can be considered as an extension of the surface transpiration approach. The velocity correction is applied throughout the flow field as opposed to only on the surface as in the surface transpiration method. Mathematically, the field velocity approach can be explained by considering the velocity field $\mathbf{V}$ in the computational domain. It can be written as:

$$
\mathbf{V}=\left(u-x_{\tau}\right) \mathbf{i}+\left(v-y_{\tau}\right) \mathbf{j}+\left(w-z_{\tau}\right) \mathbf{k}
$$

Where $u, v$ and $w$ are the components of the velocity along the coordinates directions and $x_{\tau}, y_{\tau}$, and $z_{\tau}$ are the grid time metric components. For the flow over a stationary wing these components are zero. Let a gust be represented by velocity ${ }^{w_{g}}$ along the $Z$ direction (Fig.8). Thus, the velocity field becomes:

$$
\mathbf{V}=\left(u-x_{\tau}\right) \mathbf{i}+\left(v-y_{\tau}\right) \mathbf{j}+\left(w-z_{\tau}+w_{g}\right) \mathbf{k}
$$

After $N$ iterations, the solution may not be the final equilibrium point between elastic and aerodynamic forces dependent on the assumed structural configuration, but it doesn't matter.

The Outer-most Level. This level simply is executed until overall convergence is reached. 


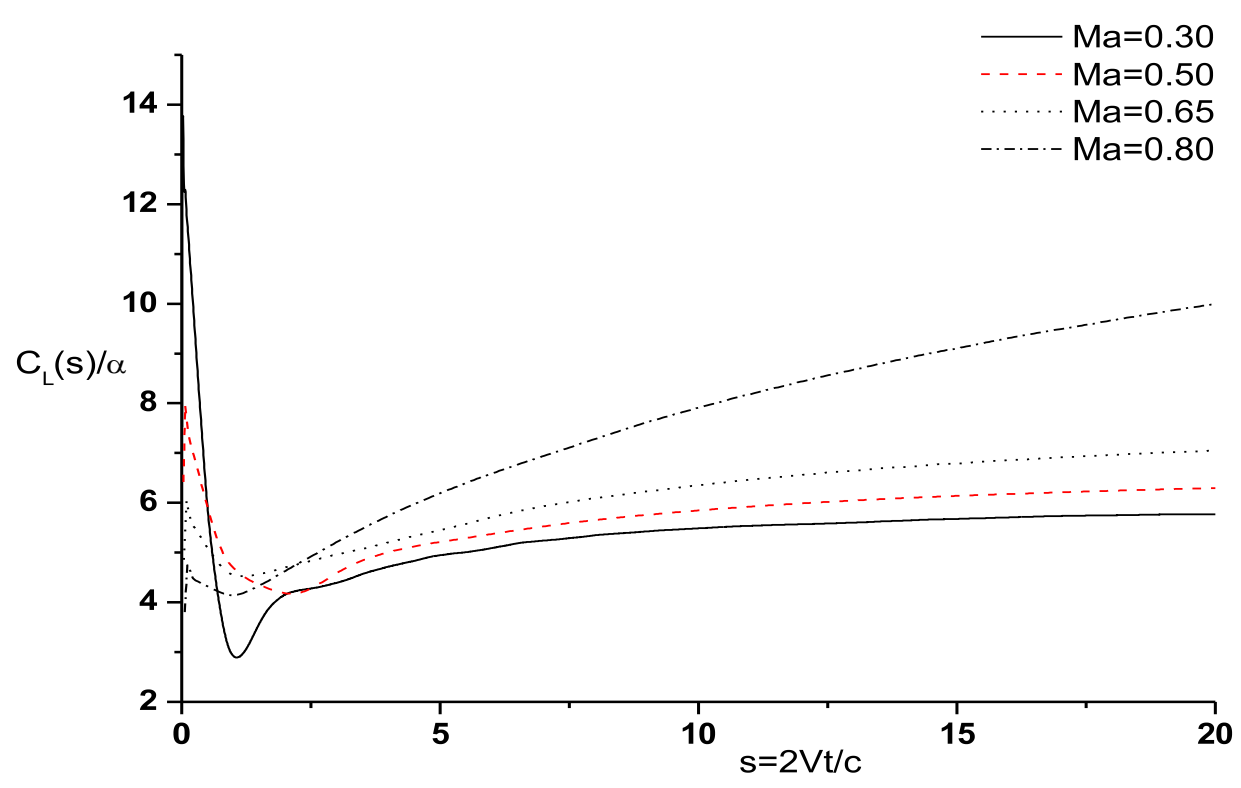

(a) Lift coefficients

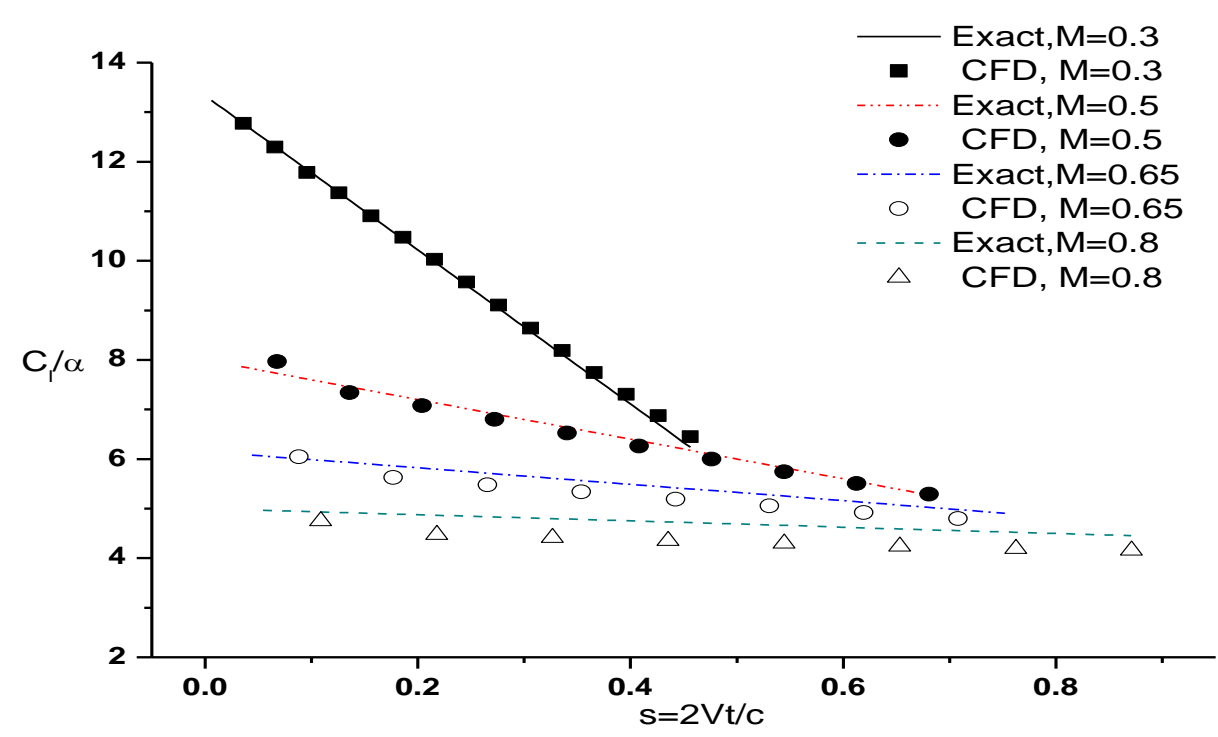

(b) Comparison with exact results

Fig. 9: Results of the sharp-edge gust response using CFD

Fig.9(a) shows the computed coefficients of lift for the sharp-edge gust response, as a function of the nondimensional aerodynamic time $s=2 V_{\infty} / c$.

Fig.9(b) shows a comparison of the computed and exact results at small times. It can be seen that the agreement is excellent. The unsteady flow computation and gust response analysis by CFD adopted here are validated by these computations.

\section{Numerical Model and Results}

\subsection{Aircraft Model}

Because it is hard to find the structural data of a fle xib le vehicle, a wing-body-tail aircraft configuration with slender wings (aspect ratio 33.33) designed by the author, as shown in Fig.10, which may not be reasonable, is selected as a test case for the numerical simulation. The total length of the fuselage is $30 \mathrm{~m}$. The detail geometric properties and mass properties are included in Table 1 and Table 2, respectively. 


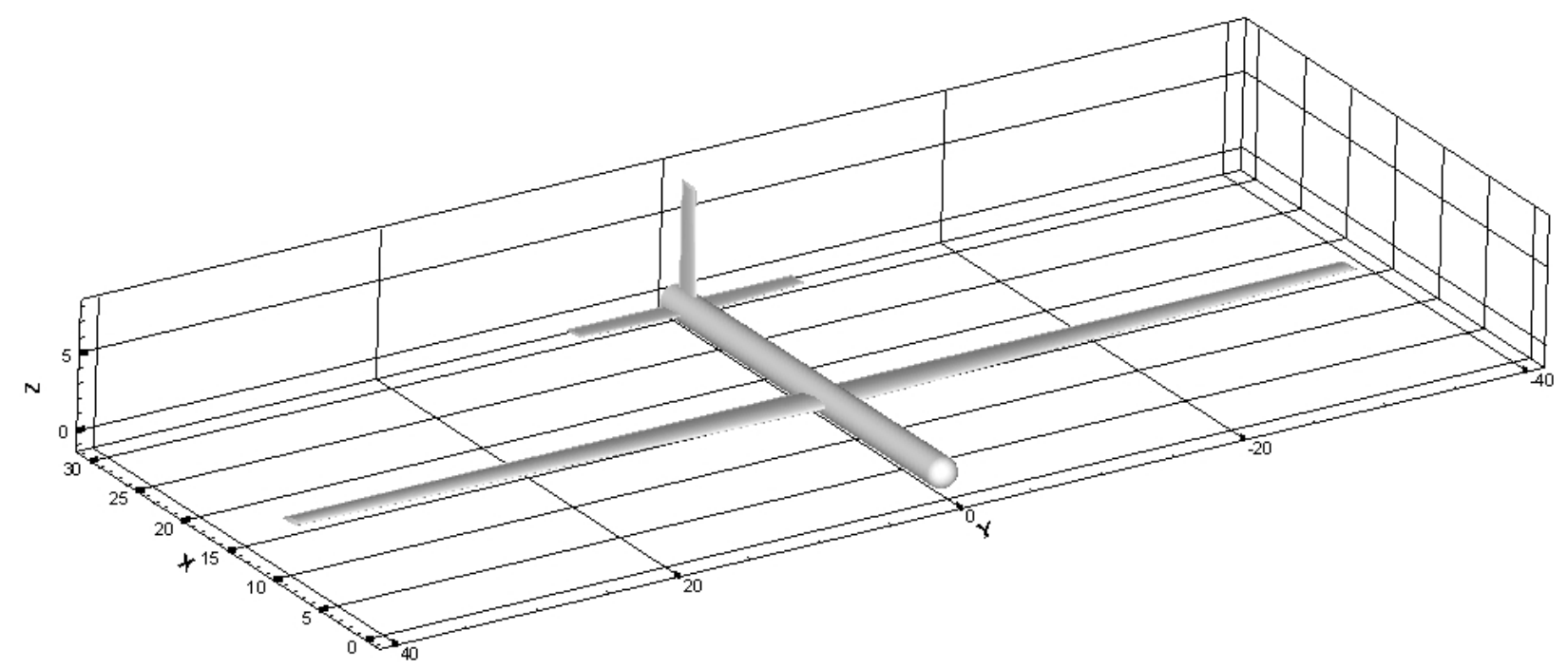

Fig. 10: Slender-wing HALE aircraft configuration

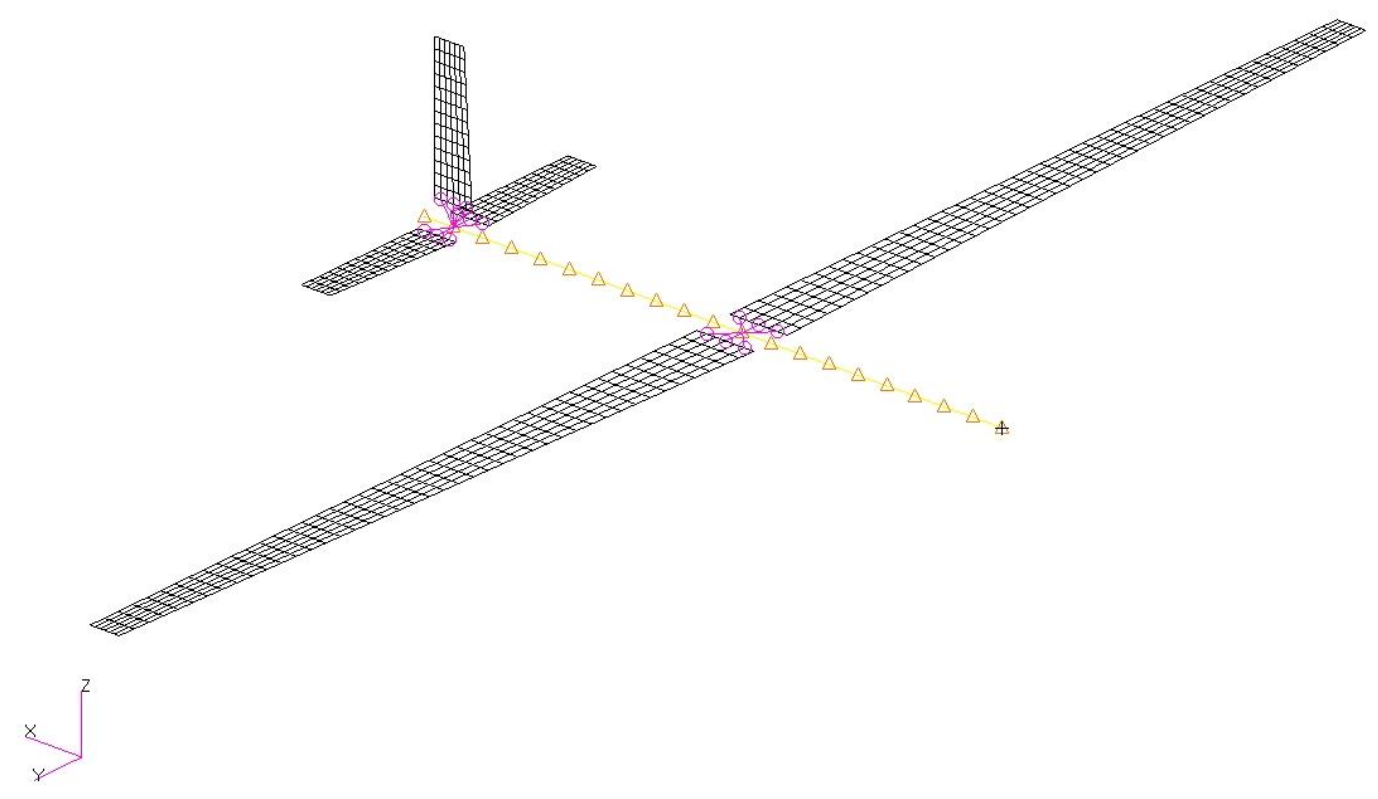

Fig. 11: Structural model of the aircraft

Table 1: Geometry of The Slender-Wing Model

\begin{tabular}{|l|c|c|c|}
\hline & Wing & HTP & VTP \\
\hline Span & $75 \mathrm{~m}$ & $16 \mathrm{~m}$ & $8 \mathrm{~m}$ \\
\hline Root Chord & $3.0 \mathrm{~m}$ & $2.0 \mathrm{~m}$ & $1.0 \mathrm{~m}$ \\
\hline Tip Chord & $1.5 \mathrm{~m}$ & $1.2 \mathrm{~m}$ & NACA 0012 \\
\hline Airfoil & NACA 4415 & NACA 0012 & $0^{\circ}$ \\
\hline Incidence Angle & $2.0^{\circ}$ & $2.0^{\circ}$ & Wing-to-HTTP: $15 \mathrm{~m}$ \\
\hline \multicolumn{4}{|c|}{ Wing-to-Nose: $12 \mathrm{~m}$} \\
\hline
\end{tabular}


Table 2: Inetia Properities

\begin{tabular}{|l|l|}
\hline Centerof mass & $x_{c}=13.808743 \mathrm{~m}, y_{c}=0.0 \mathrm{~m}, z_{c}=0.021649 \mathrm{~m}$ \\
\hline Pitching moment of inertia & $I_{y y}=8.17175 \times 10^{5} \mathrm{~kg} \cdot \mathrm{m}^{2}$ \\
\hline Total mass & $12091.605469 \mathrm{~kg}$ \\
\hline
\end{tabular}

In the modeling of the FEM, the fuselage is modeled as a beam. The wing, the elevators and the vertical rudder are modeled as shell element, and contacted with the fuselage by MPC, the FE model is presented in Fig.11.

As an example for the coupled simulation, a oneminus-cosine gust has been defined. In detail, to reduce the complexity of the problem, a symmetric flight condition has been considered. The reference flight condition is Mach 0.5 at sea level. The simulation has been performed both with a rigid and with an elastic model. One goal of the simulations is to assess of the differences between the two approaches, most notably to see whether an elastic model has an influence on the prediction of the aerodynamic loads and the flight properties.

An incrementally complex problem has been considered:

a) The aircraft is first trimmed in a desired flight condition, by computing the pitch, elevator settings and the structure deformation for level flight; b) A one-minus-cosine gust starting from the trimmed flight condition is considered, with the aircraft constrained by a revolute joint about the pitch axis.

c) The same gust velocity profile is considered, with the aircraft constrained only by symmetry constraints (vertical and longitudinal displacement, pitch rotation); Thrust is assumed to be an applied force on the aircraft center of gravity that compensates the horizontal forces on the trimmed condition.

\subsection{Trim Results}

In case of a stationary, straight symmetrical flight, all asymmetric equations of motion reduce to zero. Two cases were considered, the rigid-run and elastic-run. In this work, the iteration number $M=N=5$, the ending conditions is $\left|x^{(n+1)}-x^{(n)}\right| \leq 1.0 \times 10^{-6}$.For the rigid-run, Fig.12 shows the convergence history of the trim problem for the rigid run.

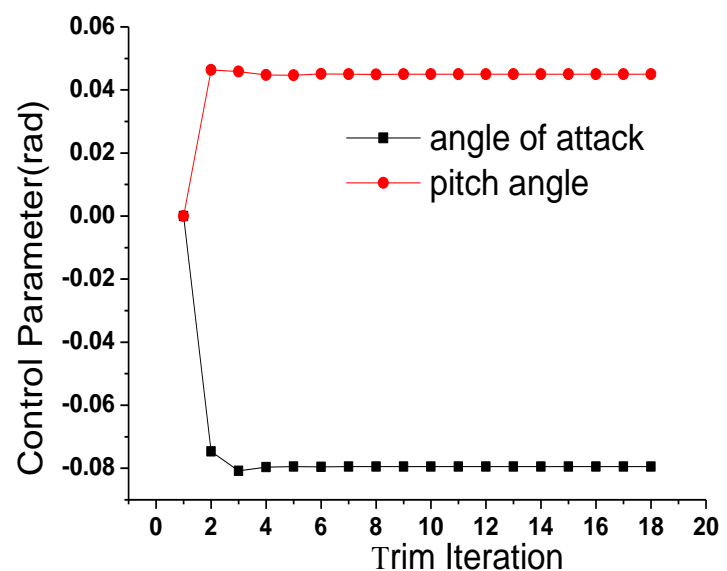

(a) Trim variables at each iteration

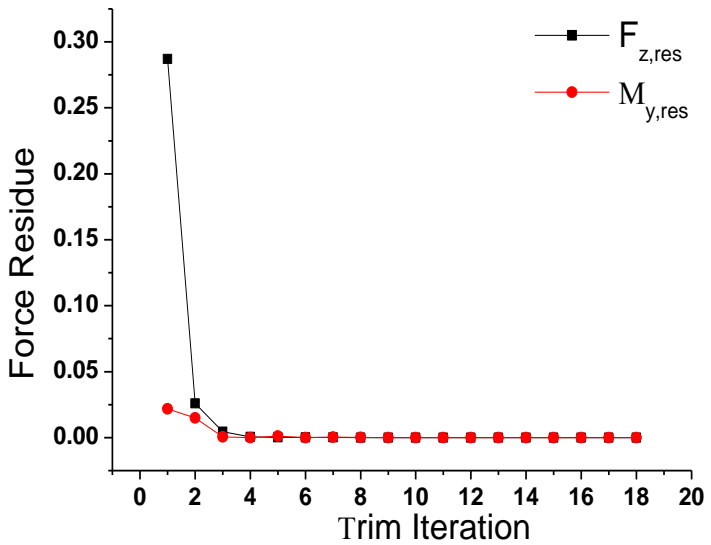

(b) Residual of the normal force and moment coefficient

Fig. 12: Convergence of the trim problem for the rigid-run

Fig. 13 presents the convergence of the trim problem of the elastic-run. Fig.13 (a) briefly reports the pitch angle $\psi$ and elevator deflection $\delta_{e}$ at trim iterations, Fig. 13 (b) shows the value of modal displacements at each iteration for the elastic solution. Table 3 summarizes the trim states of the rigid-run and elasticrun, which indicates that the trim states are nearly unchangeable with or without the consideration of the structural deformation due to the stronger structural mass and rigidity of the aircraft. 


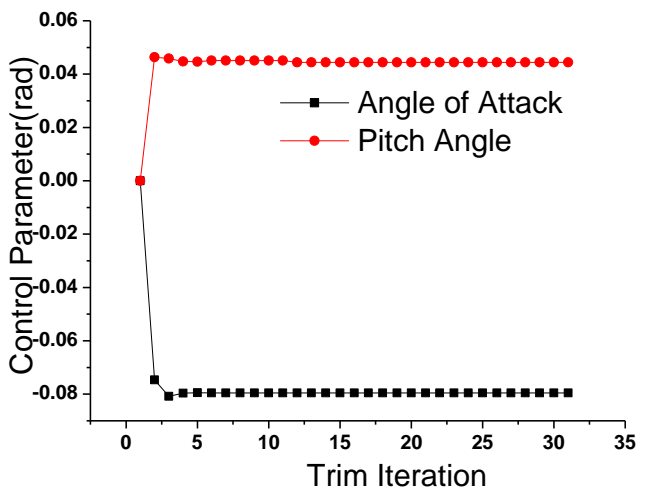

(a) Trim variables at each iteration

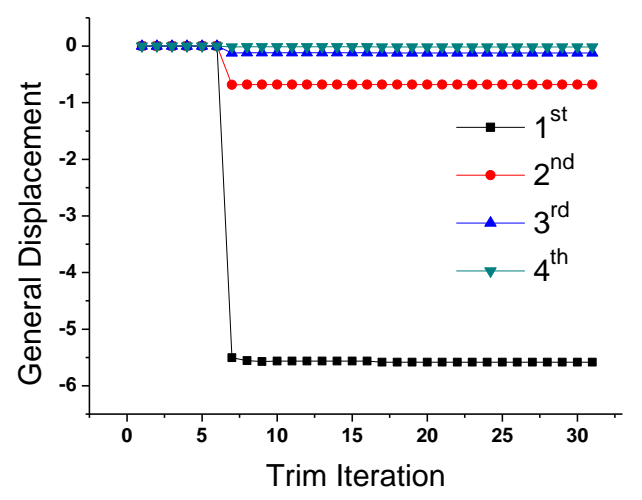

(b) Values at each trim step iteration

Fig. 13: Convergence of the trim problem for the elastic-run

Table 3: Trim States Comparsion

\begin{tabular}{|c|c|c|}
\hline & $\psi / \mathrm{rad}$ & $\delta_{e} / \mathrm{rad}$ \\
\hline Rigid-run & -0.079497 & 0.045009 \\
\hline Elastic-run & -0.079528 & 0.0443999 \\
\hline
\end{tabular}

Table 4: Trim States Comparsion

\begin{tabular}{|c|c|c|c|c|}
\hline \multirow{2}{*}{} & \multicolumn{4}{|c|}{ General displacement } \\
\cline { 2 - 5 } & $1^{\text {st }}$ & $2^{\text {nd }}$ & $3^{\text {rd }}$ & $4^{\text {th }}$ \\
\hline Rigid & 0 & 0 & 0 & 0 \\
\hline Elastic & -5.58316 & -0.680013 & -0.120040 & -0.0151869 \\
\hline
\end{tabular}

The final deformed shape is depicted in Fig.14 (a). Fig.14 (b) shows the difference in chord-wise Mach distribution for the rigid and deformable trim configurations.

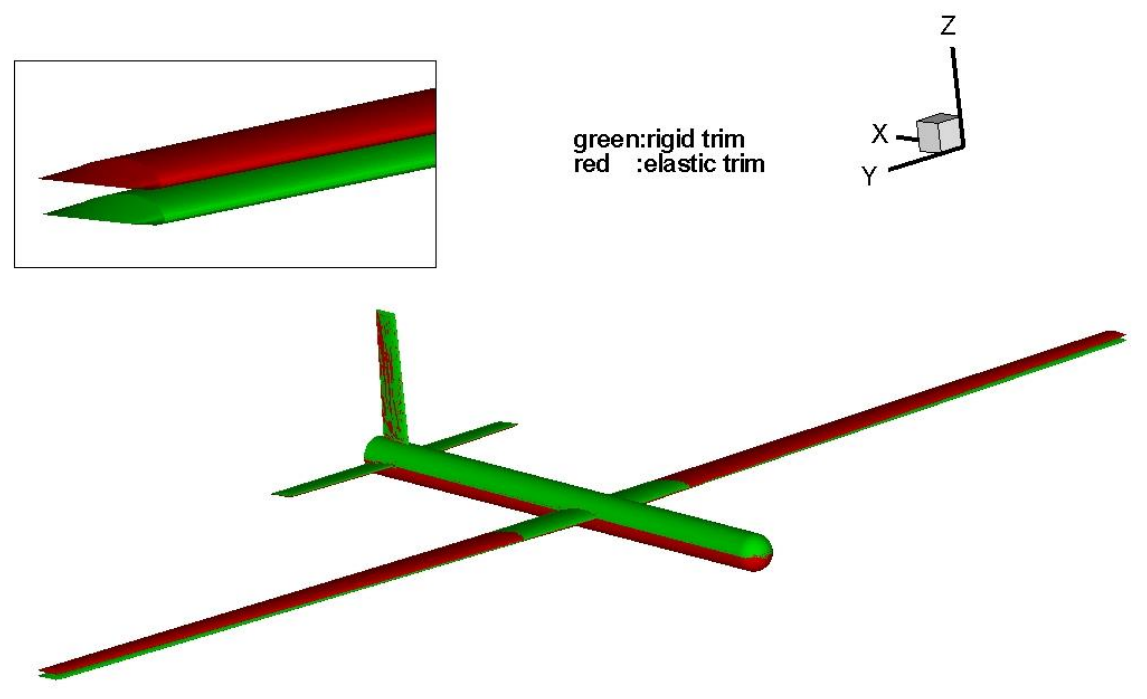

(a) Final deformed shape 


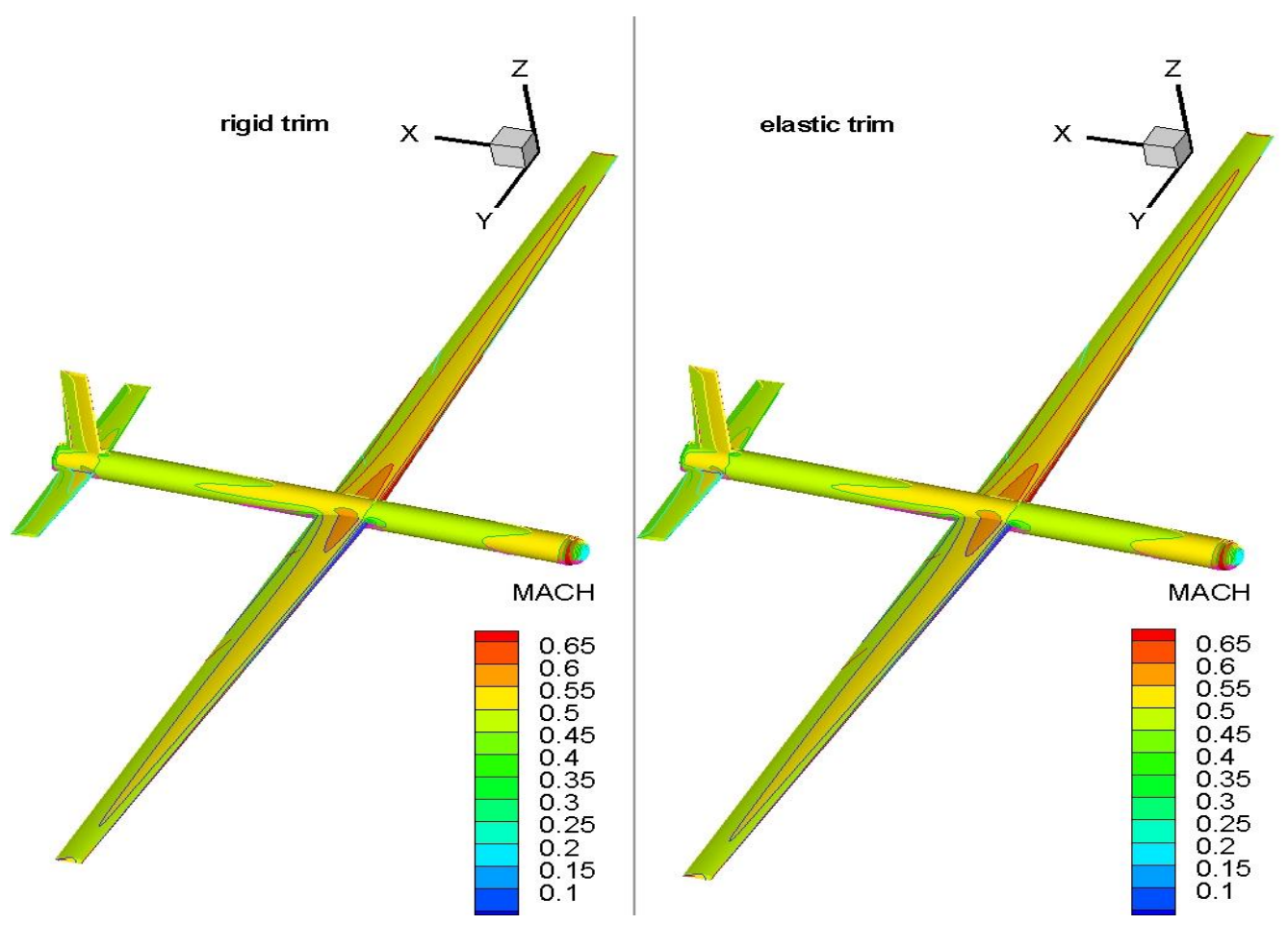

(b) Mach distribution at trim state

Fig. 14: Comparison of trimmed state between the rigid-run and elastic-run

\subsection{Free-to-pitch Simulation}

In general, gust disturbance is stochastic. In this paper, the gust model is simplified as a discrete gust having one-minus-cosine velocity profile, namely,

$$
w_{g}=\frac{1}{2} w_{0}[1-\cos (2 \pi t / 2 T)]
$$

$w_{0}$ is the design cruise gust velocity. Before and after the discrete gust pulse, there is no gust flow perturbation. The velocity profile is shown in Fig.15.
The response of the pitch angle and its' rate are shown in Fig.16 for a rigid and elastic aircraft. The initial pitch is completely recovered after the pulse response is removed. This analysis shows that the 'short-period' (pitch) motion of the aircraft is stable. The time histories of load coefficients of lift, drag, pitching moment are shown is Fig.17. Fig.18 shows the $C_{m}$ history as a function of angle of attack, which indicates that although the rigid-run and the elastic-run both recovered to the equilibrium state, the time history and hysteresis effect are still different.

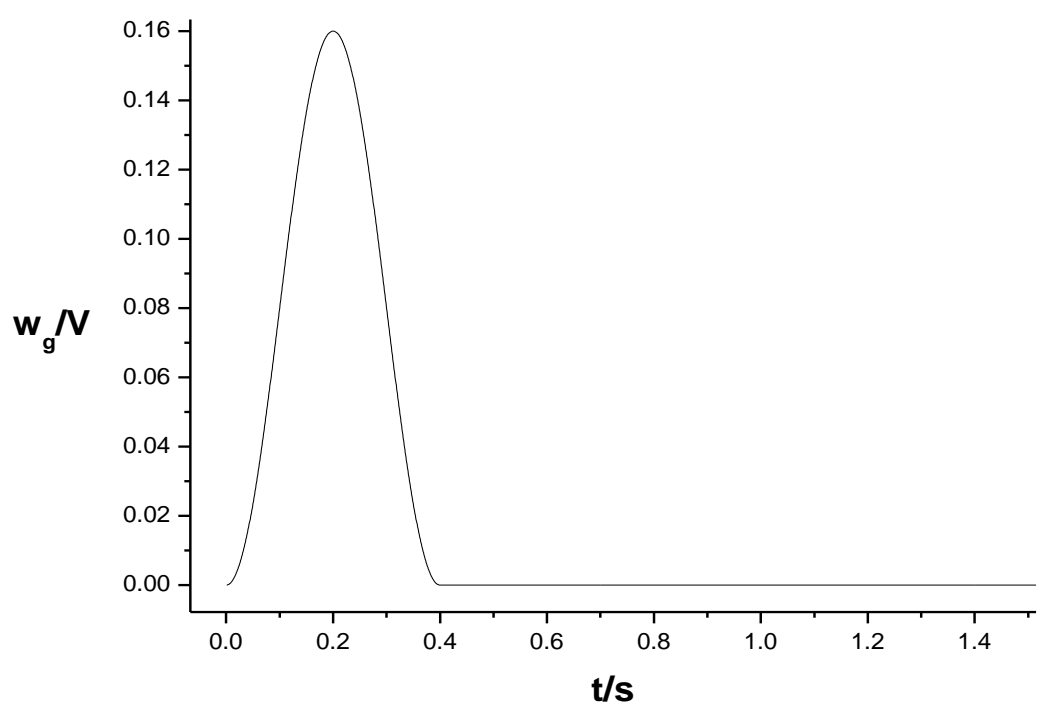

Fig. 15: Time variation of gust speed 

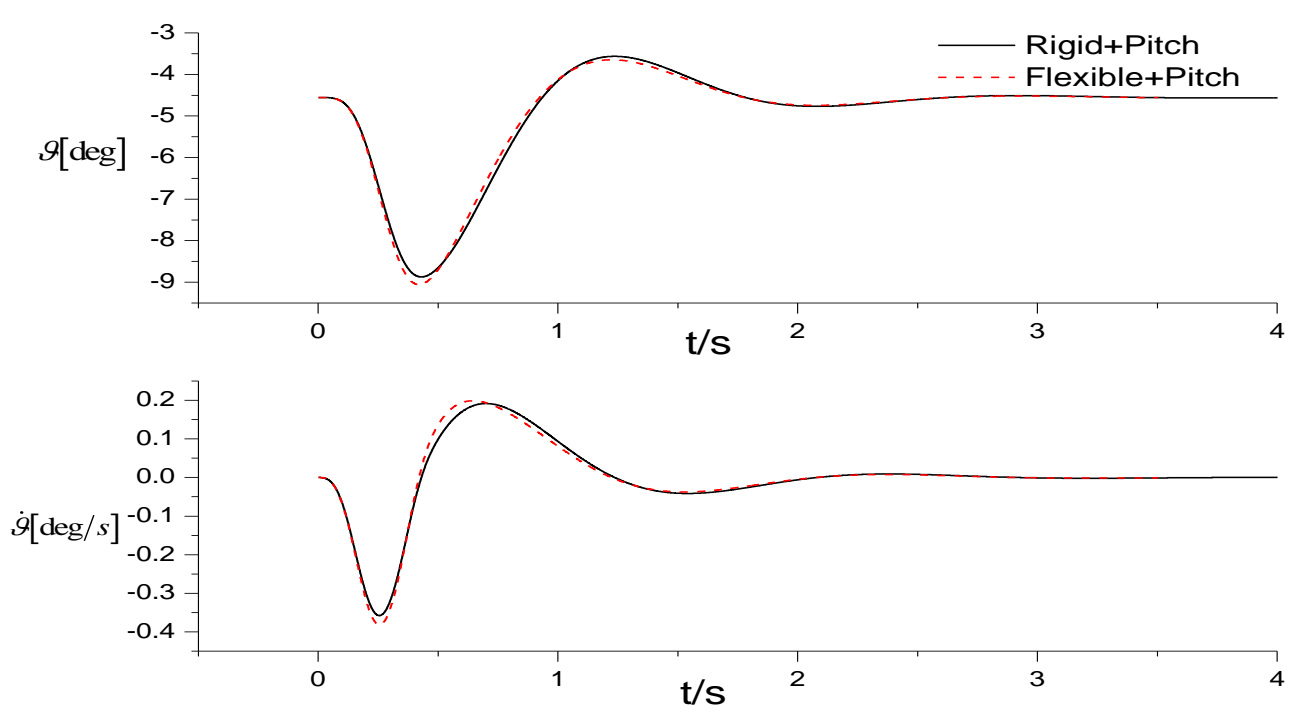

Fig. 16: Pitch rotation and rate

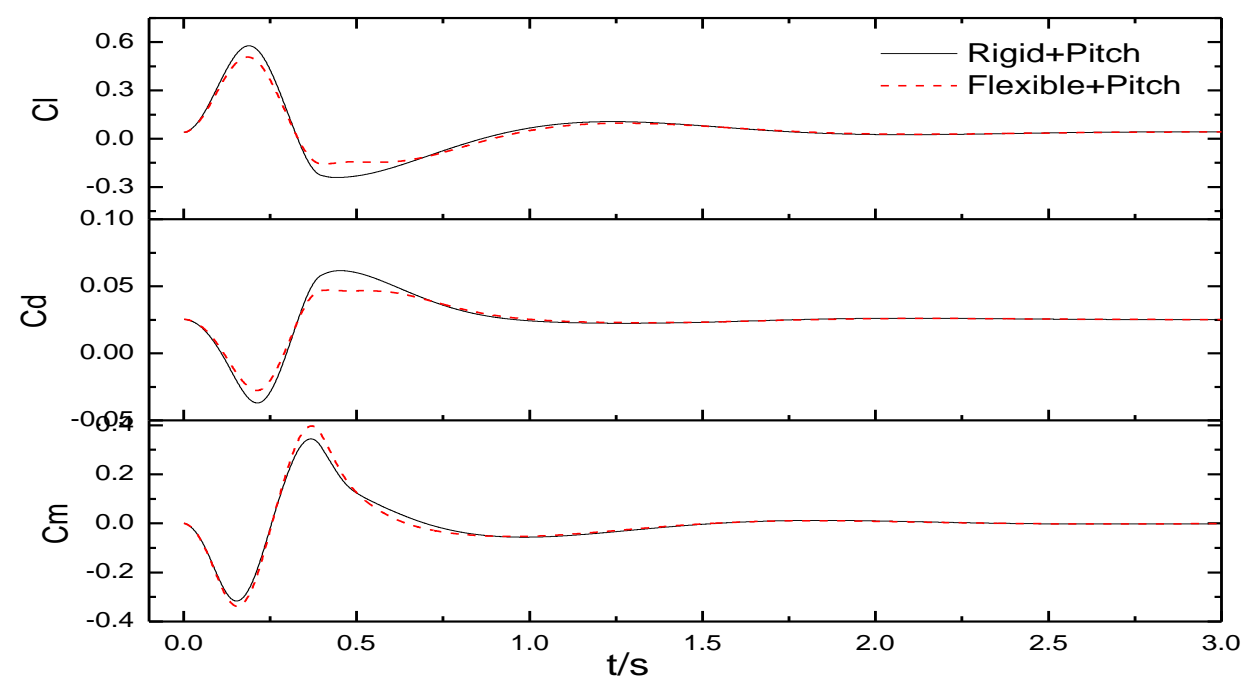

Fig. 17: Time histories of the coefficients of lift, drag and pitching moment

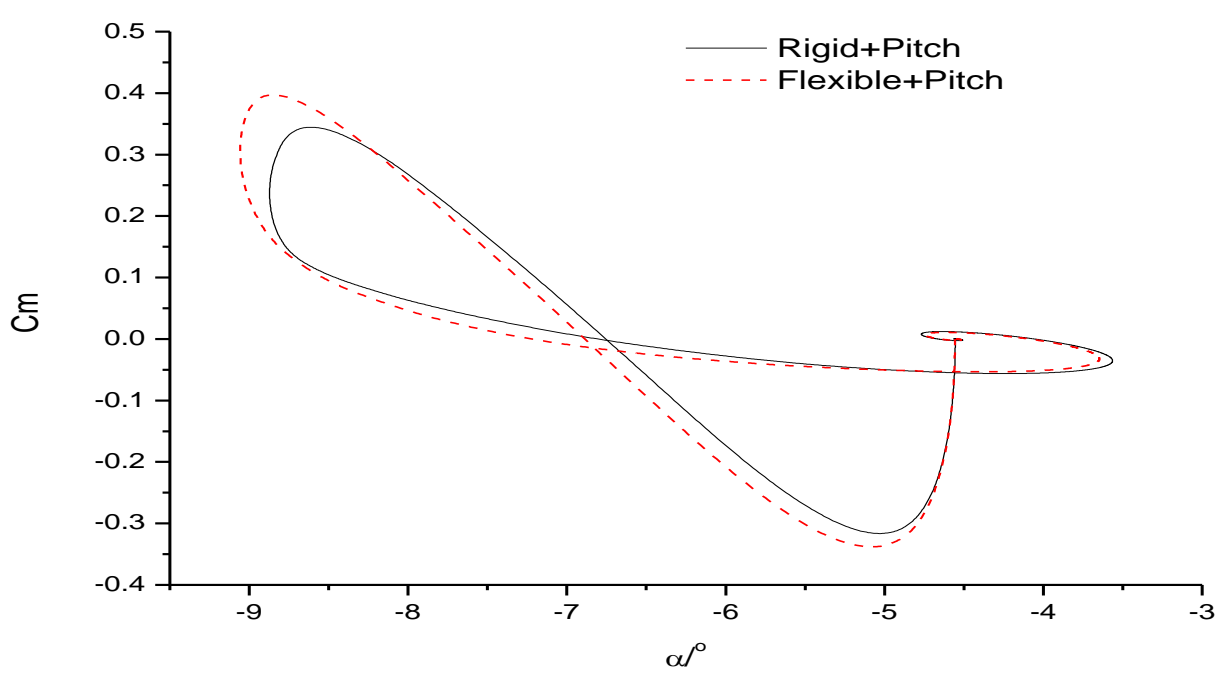

Fig. 18: Pitching moment history as a function of angle of attack 


\subsection{Free-Flying of the Longitudinal Flight}

With the same gust velocity profile, the displacement in translations, plunging and the angular displacement in pitch are shown in Fig.19. As illustrated by these figures, the free model ends up in an almost steady ascent. The motion of the aircraft should evolve into a 'phugoid' transient (altitude/horizontal speed oscillation). However, the duration of the period is much longer than what one would expect to capture with this type of analysis.
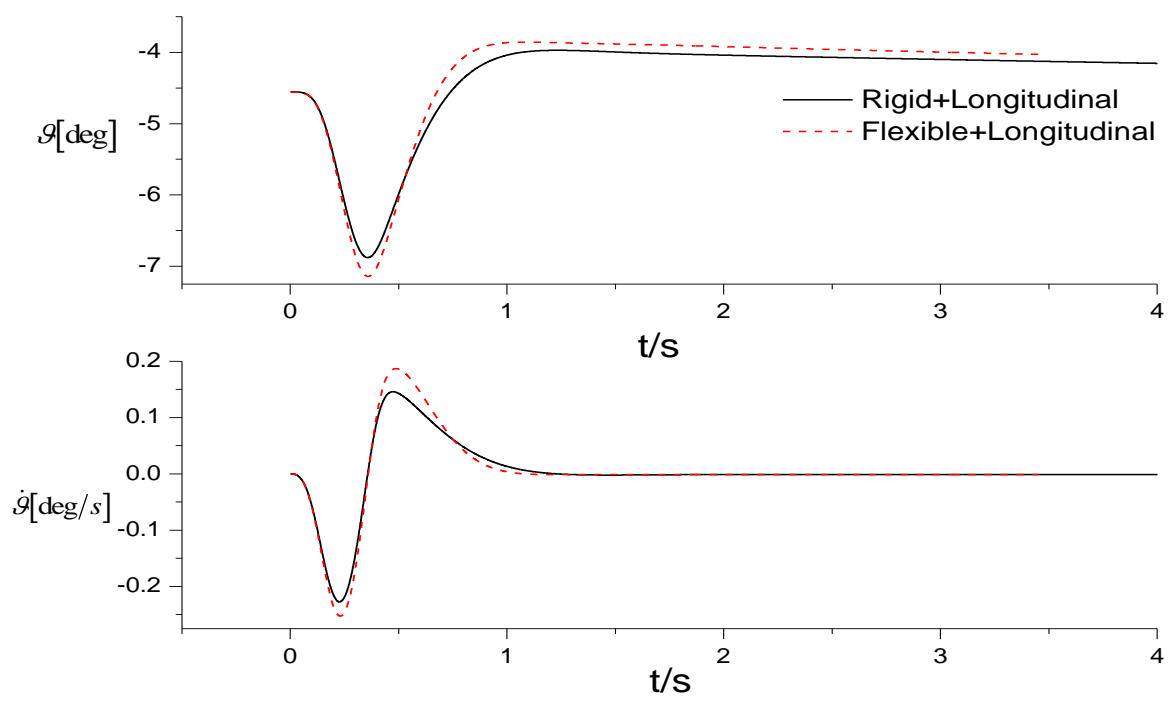

(a) Pitch rotation and rate
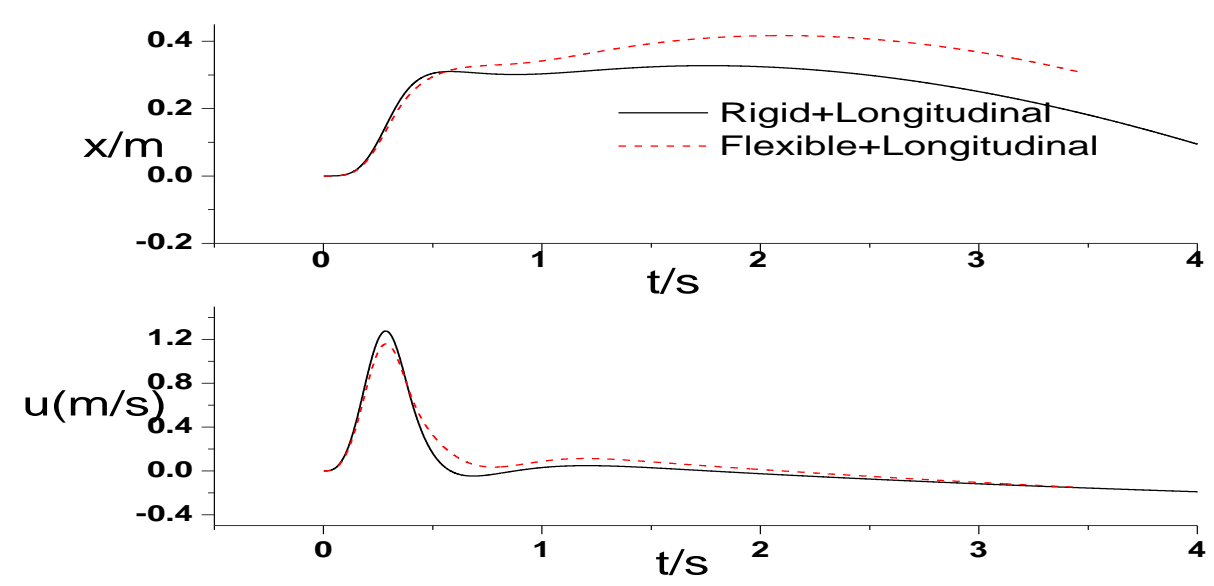

(b). Solution along $\mathrm{x}$ axis
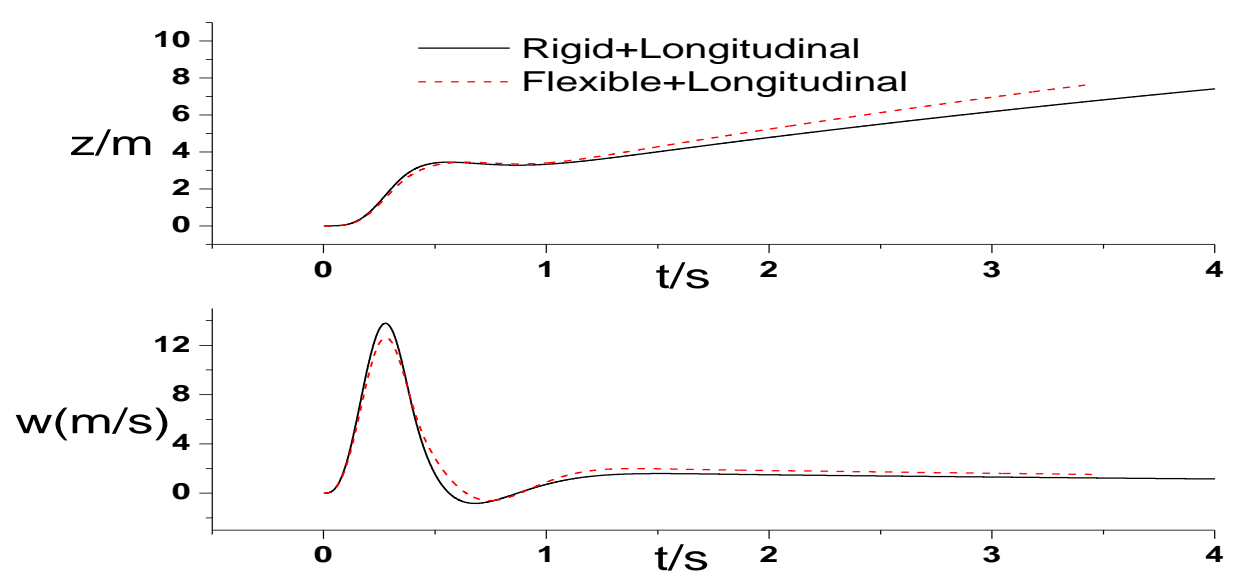

(c). Solution along $\mathrm{z}$ axis

Fig.19: Response for the free flying case 
Fig. 20 shows the time histories of the coefficients lift, drag and pitching moment. Fig.21 gives the time histories of structural deformation of the generalized displacements. The structure deformation occurs mainly in the first two modes, which corresponds to the first and second bending modes of the wing. After the pulse response, structural deformation oscillates to revert to the original equilibrium state for the free to pitch, when take into account of the plunging and translation, the structure deformation oscillates to a new equilibrium state faster. Fig.22 shows the time history of pitching moment of inertia, it varies with the structure deformation, and indicates that the mass distribution of the aircraft.

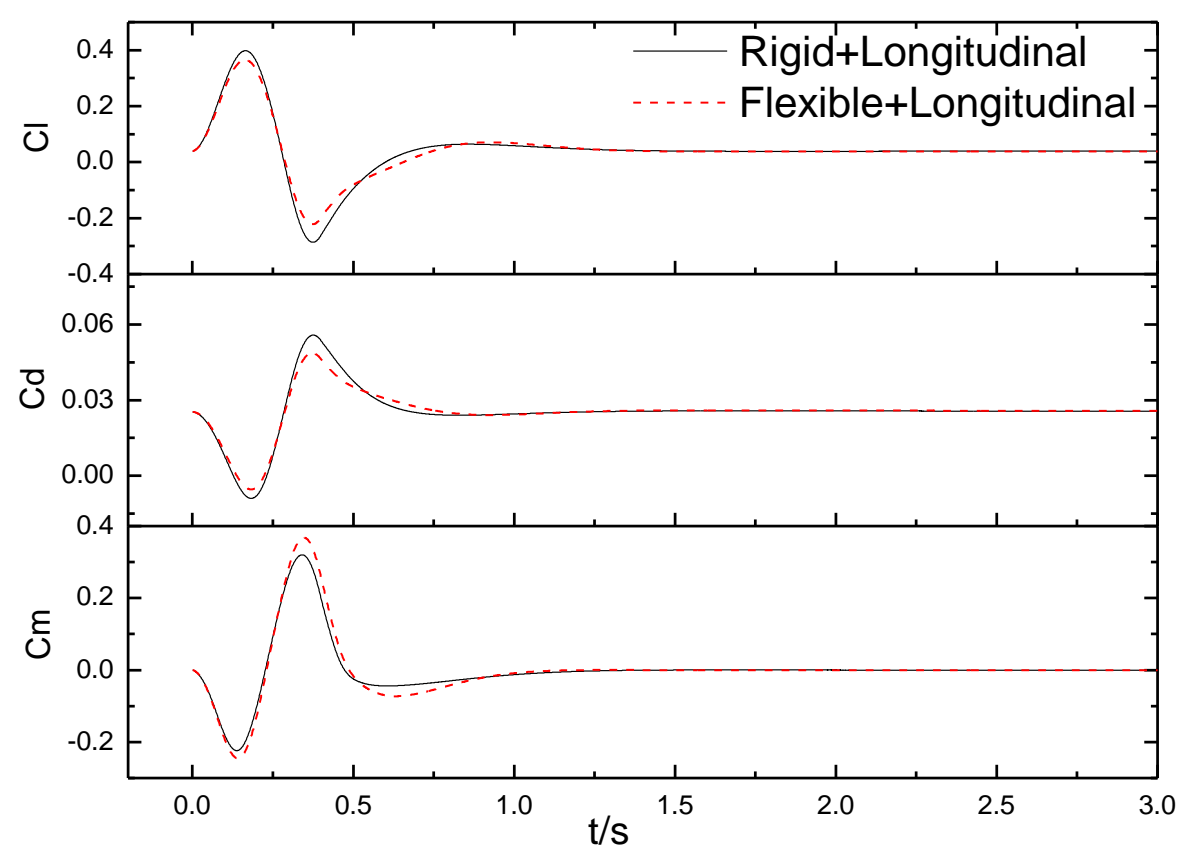

Fig. 20: Time histories of the coefficients of lift, drag and pitching moment
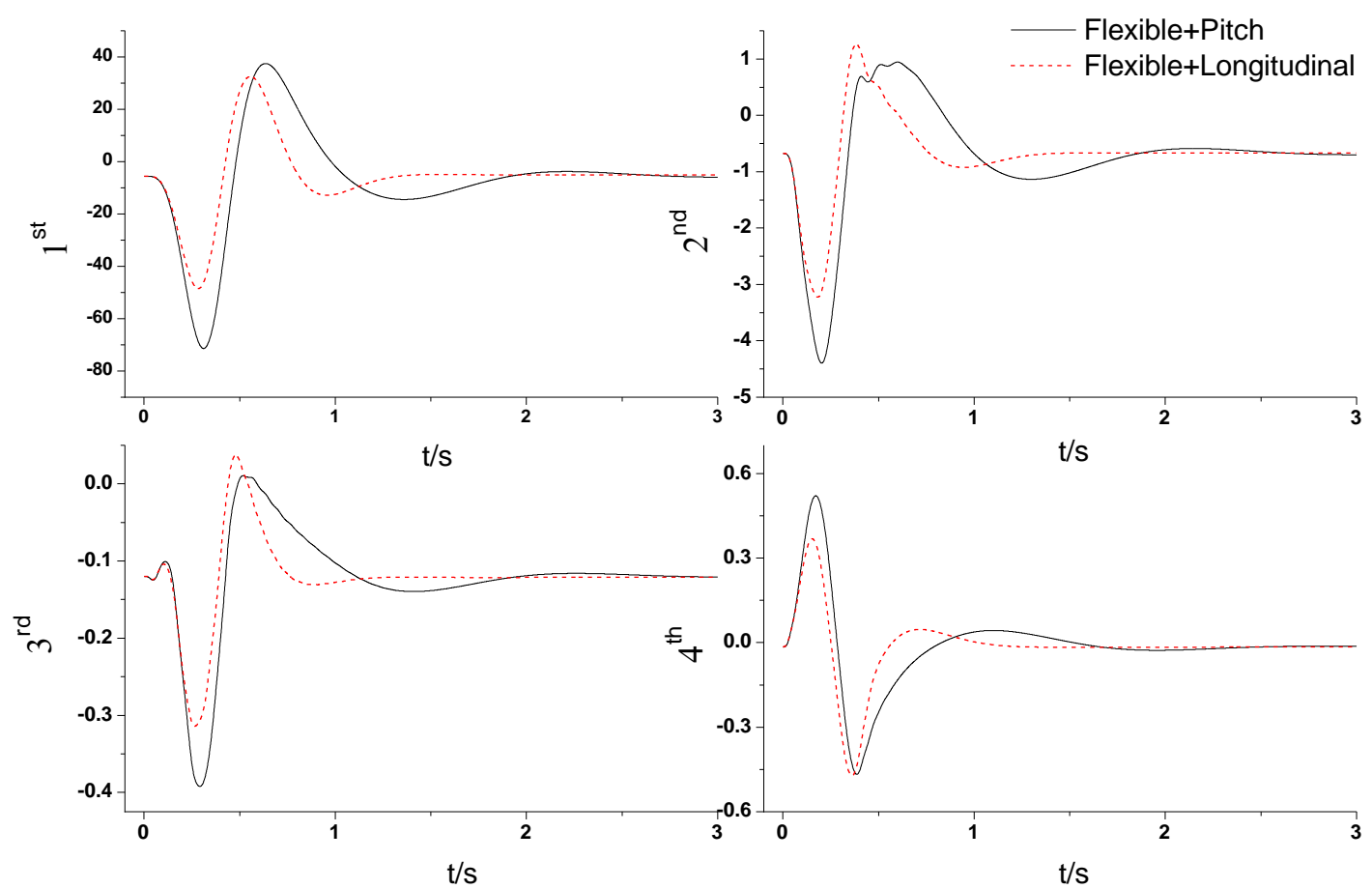

Fig. 21: Time histories of structural deformation of the first four symmetric modes for the flexible configuration 


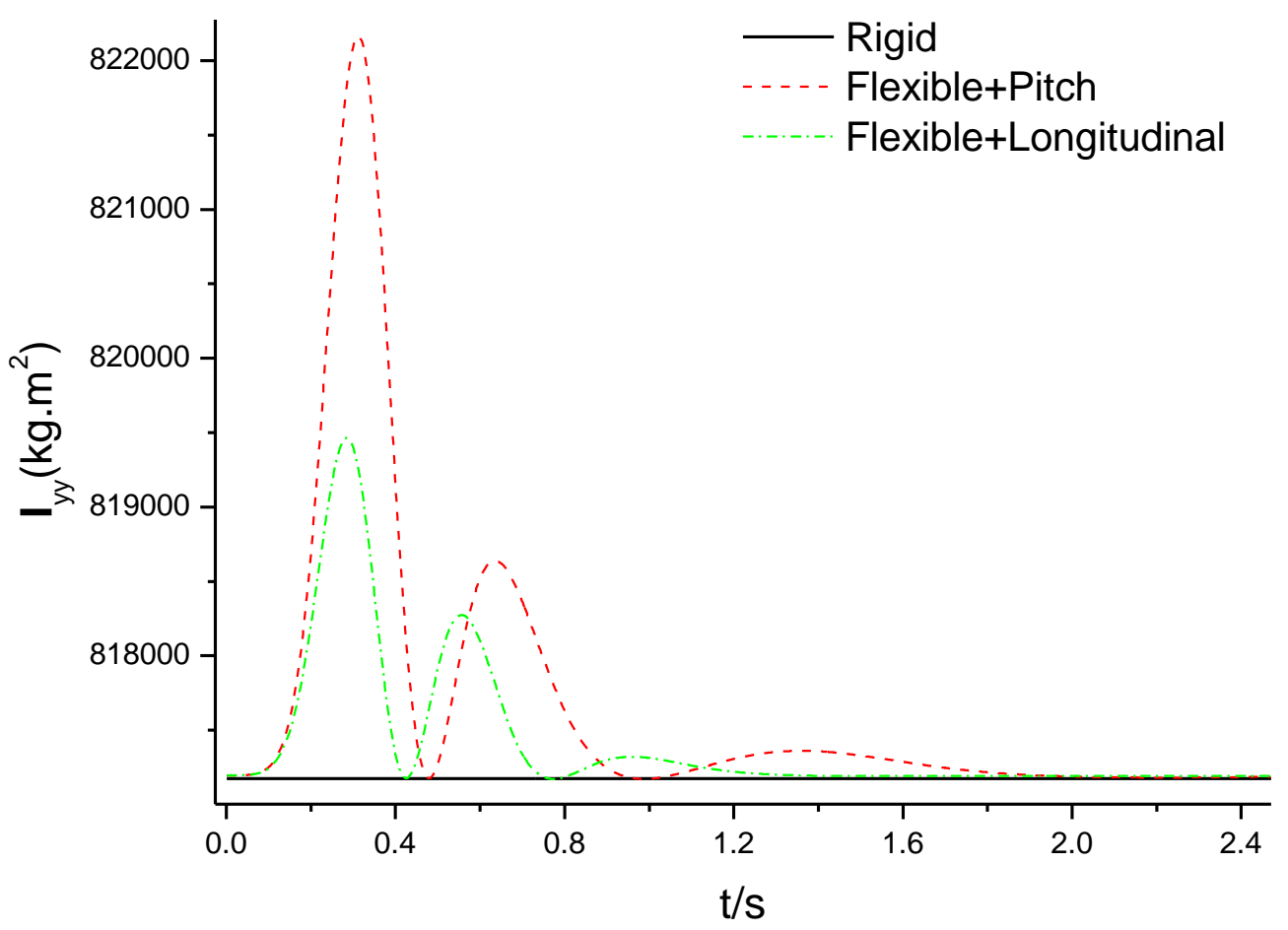

Fig. 22: Time history of the pitching moment of inertia for the aircraft

\section{Conclusion}

In the present paper, a multidisciplinary coupled numerical tool to simulate detailed aircraft models undergoing arbitrary free flight motion in the time domain was illustrated. The simulation tool combines time-accurate aerodynamic, aeroelastic and flightmechanic calculations to achieve this objective. This type of problems requires the use of sophisticate fluiddynamics models, in order to capture the relevant dynamic effects of unsteady transonic flow, including aerodynamic loads that depend on unsteady compressibility effects.

The numerical tool uses an advanced coupled computational fluid dynamics (CFD), computational structural dynamics (CSD), and computational flight mechanics (CFM) technique that provides for a gird motion capability to fly an elastic aircraft on the supercomputers.

A novel method for CFD-based maneuver trim analysis was presented. The method belongs to the category of closed coupled aeroelastic analyses, in which the flow analysis, elastic deformations, and trim computations are performed within the CFD code, within a single run. The results of trim and transient analysis applied to a slender wing HALE aircraft show the soundness of the proposed procedure.

\section{Acknowledgments}

The authors would like to thank the anonymous reviewers for their careful reading of this paper and for their helpful comments. This work was supported by the National Natural Science Foundation of China under grant no. 90816008 and Doctoral Fund of Ministry of Education of China under grant no.20070699054.

\section{References}

[1] Anon., Gust and Turbulence Loads. Code of Federal Regulations, Aeronautics and Space, Part 25.341, National Archives and Records Admin istration, Office of the Federal Reg ister, Jan. 2003

[2] Guowei Yang, Shigeru Obayashi. Numerical Analyses of Discrete Gust Response for an Aircraft. Journal of Aircraft, 2004, 41(6): 1353-1359

[3] Perry, B. III, Pototzky, A.S., and Woods, J.A., NASA Investigation of a Claimed 'Overlap' Between Two Gust Response Analysis Methods. Journal of Aircraft, 1990, 27(7): 605-611

[4] Scott, R.C., Potozky, A.S., and Perry, B., III. Computational of Maximized Gust Loads for Nonlinear A ircraft Using Matched-Filter-Based Schemes. Journal of Aircraft, 1993, 30(5):763-768 
[5] Lee, Y.N., Lan, C.E., Analysis of Random Response with Nonlinear Unsteady Aerodynamics. Journal of Aircraft, 2000, 38(8):1305-1312

[6] Flávio J Silvestre, Pedro Paglione. Dynamics and Control of a Flexible Aircraft. AIAA 2008-6876

[7] Patil M. J., Hodges, D.H. and E.S.Cesnik. Nonlinear Aeroelasticity and Flight Dynamics of High-Altitude Long Endurance Aircraft. Journal of Aircraft, 2001,38(1):88-94

[8] Shear C.M. and Cesnik C.E.S. Nonlinear Flight Dynamics of Very Flexible Aircraft. AIAA Atmospheric Flight Mechanics Conference and Exhibit, San Francisco, CA, Aug.15-18, 2005

[9] Weihua $\mathrm{Su}$, Carlos E.S.Cesnik. Nonlinear Aeroelasticity of a Very Flexible Blended-WingBody Aircraft. AIAA 2009-2402

[10] Weihua Su, Carlos E.S.Cesnik. Dynamic Response of Highly Flexible Flying Wings. AIAA 20061636

[11] Thoms E. Noll, John M. Brown, Marla E. PerezDavis. Investigation of the Helios Prototype Aircraft Mishap. http://www.nasa.gov/pdf/64317main_helios.pdf.

[12] Luca Cavagna, Pierangelo Masarati, Giuseppe Quaranta. Simulation of Maneuvering Flexible Aircraft by Coupled Multibody/CFD. Multibody Dynamic 2009, ECCOMAS Thematic Conference.

[13] Martin R. Waszak and David K. Schmidt. Flight Dynamics of Aeroelastic Vehicles. AIAA Journal of Aircraft, 1988, 25(6):563-571

[14] Weigang Yao. Nonlinear Aeroelastic System Simulation in Time Domain [Ph.D]. Northwestern Polytechnical University, Xi'an, China, 2009

[15] Robert L.Harder, Robert N.Desmarais . Interpolation Using Surface Splines. Journal of Aircraft, 1972,9(2):189-191

[16] Daniella E.Raveh. Maneuver Load Analysis of Overdetermined Trim Systems. Journal of A ircraft, 2008, 45(1):119-129

[17] Vasudev Parameswaran, James D.Baeder. Ind icial Aerodynamic in Compressible Flow-Direct Computational Fluid Dynamic Calculations. Journal of Aircraft, 1997, 34(1):131-133

[18] Rajnessh Singh, James D.Baeder. Direct Calculation of Three-Dimensional Indicial Lift Response Using Computational Fluid Dynamics. Journal of Aircraft, 1997, 34(4):465-471

\section{Authors' Profiles}

GUO Dong $(1986$ - ), male, xi'an, China, PhD candidate, his research directions include elastic aircraft flight dynamics and aeroelastic dynamics.

XU Min (1956-), female, JiangSu, China, Professor, supervis or for $\mathrm{Ph} . \mathrm{D}$. candidate, her research directions include elastic aircraft flight dynamics and aeroelastic dynamics.

CHEN Shilu (1920-), male, ZheJiang, China, Chinese Academy of Engineering, Professor, $\mathrm{PhD}$ candidate supervisor, his research directions include elastic aircraft flight mechanics and control.

How to cite this paper: Guo Dong, Xu Min, Chen Shilu,"Nonlinear Gust Response Analysis of Free Flexible Aircraft", International Journal of Intelligent Systems and Applications(IJISA), vol.5, no.2, pp.1-15, 2013.DOI: 10.5815/ijisa.2013.02.01 